Marquette University

e-Publications@Marquette

Biomedical Engineering Faculty Research and

Publications

Biomedical Engineering, Department of

5-1-1994

\title{
Non-Rayleigh Statistics of Ultrasonic Backscattered Echo from Tissues
}

\author{
V. Manjo Narayanan \\ Drexel University \\ Robert C. Molthen \\ Marquette University, robert.molthen@marquette.edu \\ P. M. Shankar \\ Drexel University \\ J.M. Reid \\ Drexel University
}

Published version. Published as part of the proceedings of the conference, 127th Meeting of the Acoustical Society of America, 1994: 2854. DOI. (C) 1994 Acoustical Society of America. Used with permission.

Robert Molthen was affiliated with Drexel University at the time of publication. 
2pAO12. Comparisons of ridge crest earthquake waveforms recorded on SOSUS hydrophones with ocean bottom seismometers in the near field. Anthony E. Schreiner (Cooperative Inst. for Marine Resource Studies, Oregon State Univ., 2115 S.E. OSU Dr., Newport, OR 97365) and Christopher G. Fox (NOAA/PMEL, Newport, OR 97365)

The U.S. Navy's SOSUS network of hydrophone arrays is allowing an unprecedented continuous look at oceanic seismicity. Hydrophones in the SOFAR channel are sensitive detectors of acoustic T-waves resulting from earthquakes in the ocean crust. Since Fall of 1991 a program at the NOAA Pacific Marine Environmental Laboratory has catalogued over 500 seismic events originating on the boundaries of the Pacific, Gorda, and Juan de Fuca tectonic plates. Though extremely sensitive, observations through SOFAR hydrophones have the drawback that the earthquake signal travels long distances though the ocean sound channel. The recorded signal is thus a highly distorted representation of the earthquake. There is as yet no reliably determined relationship between $\mathrm{T}$-wave strength and source magnitude, mainly be- floor into the SOFAR waveguide. We have compared earthquake and explosive events recorded in 1991 simultaneously by the SOSUS network and three small-scale arrays of ocean bottom seismographs placed near the intersection of the Gorda spreading ridge and the Blanco Transform Fault. By this effort, we are attempting to gather enough statistics on long range T-wave strength compared to local measurements to provide a rough magnitude scale.

4:55-5:00 Break

5:00-5:30

\section{PANEL DISCUSSION:}

Panel Moderator: David Gardner

Panel Members: Dennis M. Colon, Kirk Evans, Christopher G. Fox, Robert F. LaPlante, James A. Mercer, Mary P. Mosier, Harold A. Williams

\title{
TUESDAY AFTERNOON, 7 JUNE 1994 STUDENT CENTER, WEST LOUNGE, 1:15 TO 4:30 P.M.
}

\author{
Session 2pBV
}

\section{Bioresponse to Vibration and to Ultrasound and Physical Acoustics: Biomedical Ultrasound: Techniques and Cavitation Effects}

\author{
E. Carr Everbach, Chair \\ Department of Engineering, Swarthmore College, 500 College Avenue, Swarthmore, Pennsylvania 19081
}

\section{Contributed Papers}

\section{1:15}

2pBV1. Wavespace resolution in ultrasonic backscatter measurements. T. Douglas Mast and Robert C. Waag (Dept. of Elec. Eng., Univ. of Rochester, Rochester, NY 14627)

Pulse-echo measurements of ultrasonic scattering are a useful technique for characterization of scatterer properties, since the spectra of a scatterer's spatial properties can be determined from the frequencydependent backscatter coefficient. In such measurements, the spatial localization associated with transducer beam patterns and time gates causes an uncertainty in the measured spatial-frequency domain properties of the scatterer. This uncertainty is analyzed using an analytic and computational model for measurement system effects in backscatter measurements. The results indicate that wavespace resolution is limited in the direction of the scattering vector by the finite durations of the transmitted pulse and the detector time gate, being approximately proportional to the time gate durations. Resolution in the lateral direction depends mainly on the transducer aperture, increasing approximately in proportion to the aperture diameter. Smooth aperture apodization improves lateral resolution somewhat but has little effect on resolution in the direction of the scattering vector.

\section{1:30}

2pBV2. Non-Rayleigh statistics of ultrasonic backscattered echo from tissues. V. M. Narayanan (Biomed. Eng. \& Sci. Inst., Drexel Univ., Philadelphia, PA 19104), R. C. Molthen, P. M. Shankar, and J. M. Reid (Drexel Univ., Philadelphia, PA 19104)

The envelope of the backscattered signal from tissues can exhibit non-Rayleigh statistics if the number density of scatterers is small or if the variations in the scattering cross sections are random. The $K$ distribution which has been used extensively in radar, is introduced to model this non-Rayleigh behavior. The generalized $K$ distribution is extremely useful since it encompasses a wide range of distributions such as Rayleigh, Lognormal, and Rician. Computer simulations were conducted using a simple one-dimensional discrete scattering model to investigate the properties of the echo envelope. In addition to cases of low number densities, significant departures from Rayleigh statistics were seen as the scattering cross sections of the scatterers become random. The validity of this model was also tested using data from tissue mimicking phantoms. Results indicate that the density function of the envelope can be modeled by the $K$ distribution and the parameters of the $K$ distribution can provide information on the nature of the scattering region in terms of the number density of the scatterers as well as the scattering cross sections of the scatterers in the range cell. [Work was supported by NSF Grant No. BCS-9207385.]

\section{1:45}

2pBV3. Time domain acoustic absorption: A unified model for linear and nonlinear acoustics. Kenneth D. Rolt (MIT, Dept. of Ocean Eng., Cambridge, MA 02139)

Dissipation of a linear acoustic wave, at frequency $\omega_{0}$, is directly related to the pressure absorption coefficient $\alpha(\omega)$. In the viscous case, $\alpha(\omega)=\left(\frac{4}{3} \eta+\eta_{b}\right) \omega_{0}^{2} /\left(2 \rho_{0} c^{3}\right)$. For an initially $\omega_{0}$-sinusoidal largeamplitude nonlinear wave, the absorption coefficient is usually described by a linear part, and by an excess part related to the harmonics from nonlinear distortion. An alternate way to describe the absorption of a nonlinear acoustic wave is to define a total absorption coefficient. This is done in the time domain directly by considering dissipation in the momentum equation, and then proceeding in an energy balance approach. The result gives the instantaneous $\alpha$ for a given point on the wave in space without the need for spectral decomposition. For the viscous case, $\alpha(x)=\left(\frac{4}{3} \eta+\eta_{b}\right)\left|\nabla^{2} v_{\mid x_{0}}\right| /\left(2 \rho_{0} c\left|v_{0}\right|\right)$, where $v$ is the particle velocity 